\title{
Inégalités de formation lors de l'accès à l'enseignement supérieur selon le sexe et le contexte migratoire: résultats de l'analyse des situations en Suisse, en Allemagne et en France
}

\section{Dorit Griga et Andreas Hadjar}

La présente contribution porte sur le passage dans le degré tertiaire en tenant compte de l'intrication des inégalités liées au sexe et au contexte migratoire. L'étude, qui repose sur des concepts de la théorie décisionnelle, s'articule principalement sur une comparaison entre la Suisse (TREE), l'Allemagne (élèves pouvant prétendre aux études supérieures 2002 HIS) et la France (Panel d'élèves du second degré 1995). Les résultats révèlent qu'en Suisse et en Allemagne, indépendamment de l'origine sociale, les jeunes hommes appartenant à certains groupes de migrants socialement défavorisés (2e génération) affichent un taux de passage dans le supérieur plus élevé que leurs camarades nationaux. Ils mettent par ailleurs en évidence que les migrantes de deuxième génération issues de pays fortement marqués par le modèle patriarcal ne sont désavantagées ni en Suisse, ni en Allemagne, ni en France.

\section{Introduction}

À l'heure actuelle, dans nombre de pays européens, les modèles autour desquels s'articulent la participation à la formation et, partant, les chances d'accéder à une éducation supérieure évoluent (Arum et al., 2007; Breen et al., 2012). Alors que dans quasiment tous les pays européens, les inégalités de formation liées au sexe s'inversent au profit des femmes (Buchmann et al., 2008), à première vue, il semblerait que différents groupes de jeunes issus de l'immigration soient toujours désavantagés à cet égard dans les pays d'immigration européens (Heath \& Brinbaum, 2007). Il existe certes des études s'attachant à analyser individuellement les facteurs présumés d'inégalité - à l'instar du sexe et du contexte migratoire notamment en les examinant sous l'angle de l'accès à l'enseignement supérieur. Toutefois, les éventuelles interactions qui peuvent exister entre ces deux axes et leurs possibles répercussions sur la réussite des études supérieures ont rarement été approfondies (Fleischmann \& Kristen, 2011; Solga \& Becker, 2012).

La présente contribution porte donc sur les décisions prises en matière de formation lors du passage dans le degré tertiaire en tenant compte de l'intri- 
cation du sexe et du contexte migratoire. Au-delà de la thématique des inégalités en matière d'accès aux hautes écoles reposant sur ces deux axes, elle vise également à déceler d'éventuels indices suggérant que les pays d'accueil aient, ou non, développé des schémas généraux quant aux différences présidant à l'accès aux études supérieures en fonction du sexe ou du contexte migratoire, ou si chaque État - à travers son système éducatif et les conditions encadrant son marché de l'emploi - a ses propres schémas. Cette réflexion se fonde principalement sur une étude comparative de trois pays européens terres d'immigration: la Suisse, l'Allemagne et la France.

Le prochain chapitre s'ouvre sur une brève description des trois sociétés d'accueil examinées, suivi de quelques considérations théoriques au sujet des inégalités de formation sous l'angle du contexte migratoire, de l'origine sociale et du sexe. Le chapitre suivant porte, lui, sur la stratégie d'analyse adoptée et se conclut sur une synthèse des problématiques traitées. Quant aux données et aux principales caractéristiques descriptives des échantillons utilisés, elles sont détaillées dans la chapitre concernant la méthodologie. Enfin, dans les deux derniers chapitres, les résultats sont présentés, puis mis en perspective.

\section{Comparaison entre La Suisse, L'Allemagne et La France}

Alors que la Suisse et la France sont deux des terres d'accueil traditionnelles de l'Europe avec une grande partie de la population issue de l'immigration (à l'heure actuelle elle est d'environ un tiers en Suisse selon les chiffres de l'OFS pour 2013 et d'un quart en France; Algan et al., 2010), cette tendance est plus récente en Allemagne. Si la part d'étrangers y reste plus basse, elle a néanmoins progressé rapidement pour atteindre aujourd'hui le taux significatif d'un cinquième (bpb, 2012). S'agissant des conditions-cadre institutionnelles régissant le système social et le système d'enseignement secondaire, tous les pays analysés dans la présente contribution affichent des différences intéressantes, tant eu égard à la problématique du genre qu'au niveau des différences pouvant être mises sur le compte de la migration.

Les structures de formation en Suisse, en Allemagne et en France se distinguent clairement les uns des autres, ne serait-ce que par le degré de stratification caractérisant le système scolaire (Müller \& Shavit, 1998; Hadjar \& Berger, 2010). En Suisse, où le système scolaire est assez clairement stratifié, notamment dans les cantons alémaniques, la part des élèves d'une cohorte obtenant la maturité - qui donne directement accès aux études supérieures - est relativement faible (un cinquième environ). Cette filière reste très sélective en termes d'origine sociale et de contexte migratoire (Becker \& Zangger, 2013; CSRE, 2010). En Allemagne, le taux d'étudiants passant l'Abitur se situe autour d'un tiers, il est donc plus élevé qu'en Suisse (Becker, 2013). Les systèmes scolaires des Länder allemands, 
eux aussi fortement stratifiés, ont également tendance à écarter les élèves des classes ouvrières ou issus d'une certaine immigration (en provenance de Turquie p. ex.). En France en revanche, dont le système scolaire est moins marqué par cette stratification, deux-tiers d'une cohorte environ décrochent le baccalauréat (Duru-Bellat \& Kieffer, 2008). Cependant, même si l'ampleur des inégalités est plus limitée, les jeunes des milieux défavorisés ou issus de l'immigration sont aussi victimes d'inégalités en matière de formation (Vallet \& Caille, 1999).

S'agissant des alternatives aux études supérieures, on constate des différences significatives dans l'importance de la formation professionnelle. Ainsi, les systèmes éducatifs suisse et allemand se caractérisent davantage par les qualifications étant donné qu'ils accordent un poids déterminant à ces cursus (Maurice et al. 1982; Müller \& Shavit, 1998). Il en va différemment en France, où l'école dispense un savoir plus général et où la part des jeunes optant pour les formations professionnelles est faible, tandis que nombre d'étudiants entament des études supérieures. Notons toutefois que le taux d'abandon en cours d'étude est également élevé. Certes, différentes études ont confirmé la pertinence de la thèse de Müller \& Pollak (2007) - selon laquelle les enfants d'ouvriers tendent à se détourner de l'université - pour les pays valorisant les filières professionnelles (voir pour l'Allemagne notamment Becker \& Hecken, 2009a, et pour la Suisse: Becker, 2012). Il s'agit néanmoins de se demander si cette hypothèse se vérifie dans la même mesure chez les jeunes issus de l'immigration et chez les autres.

Relevons encore que les paramètres institutionnels singularisant les pays ont également un impact sur les inégalités observées entre hommes et femmes sur le marché de l'emploi et sur la rentabilité de la formation - connu sous le nom de "gender regimes» (cf. Walby et al., 2012). Alors que la Suisse et l'Allemagne sont par exemple fortement marquées par le modèle patriarcal, c'est moins le cas de la France qui favorise la conciliation entre vie privée et professionnelle (Lewis \& Ostner, 1994), ce qui lui vaut même d'être désignée comme pays au modèle du «dual-earner» (Luci, 2011). En effet, le taux de femmes travaillant à plein temps est beaucoup plus élevé en France qu'en Suisse ou en Allemagne. D'ailleurs, en France et en Allemagne, les enfants de trois ans fréquentent respectivement à $99 \%$ et $87 \%$ des structures d'accueil contre quelque $10 \%$ en Suisse (cf. données de l'OCDE pour 2008).

Les conditions d'intégration des migrants et plus particulièrement les politiques adoptées en la matière sont relativement proches dans les trois pays étudiés, si bien que leurs scores au migrant integration policy index (MIPEX, British Council \& Migration Policy Group, 2013) se situent tous autour de la moyenne. Avec ses 57 points sur un total possible de 100, la France affiche le meilleur classement, devant l'Allemagne et ses 51 points. Quant à la Suisse, ses 43 points la placent au-dessous de la moyenne, autrement dit les migrants y sont plus souvent victimes de discriminations. 


\section{Cadre théorique}

Du point de vue de la recherche sur les inégalités relevant de la sociologie de l'éducation, on peut appréhender la question de savoir si les personnes pouvant prétendre à des études du degré tertiaire s'engagent ou non dans cette voie à l'aide des concepts de la théorie décisionnelle. Entrent ainsi dans le champ de la réflexion les effets secondaires tels que les a décrits Raymond Boudon (1974), derrière lesquels se cachent des évaluations sur la probabilité de succès, les coûts et les gains estimés des cursus de formation en général et des études supérieures en particulier, différentes d'un groupe social à l'autre ainsi que des appréciations du rapport coût-utilité des différentes options en termes de formation fondées sur le motif du maintien du statut (Erikson \& Jonsson, 1996; Esser, 1999; Becker \& Hecken, 2009b).

Si l'on s'intéresse dans un premier temps aux axes d'inégalité que sont l'origine sociale et le contexte migratoire, nombre d'études empiriques (Heath \& Brinbaum, 2007; pour la Suisse: Beck et al., 2010; Meyer, 2003; pour l'Allemagne: Kristen \& Granato, 2007; pour la France: Vallet \& Caille, 1996, 1999; Simon, 2003) montrent qu'une grande partie des handicaps relevant de la migration dans le système éducatif est à mettre sur le compte de l'origine sociale. S'agissant du recoupement de différences liées au contexte migratoire et à l'origine sociale, plusieurs études ont mis en évidence que lors du passage aux études supérieures, la probabilité que les groupes de migrants choisissent cette voie - indépendamment de leur origine sociale et de leurs résultats scolaires est même supérieure à celle du reste des élèves (Kristen et al., 2008; Jackson $e t$ al., 2012; Griga, 2014). Pour expliquer ces différences, les chercheurs en sociologie de l'éducation renvoient aujourd'hui pour l'essentiel aux aspirations plus élevées que nourrissent les jeunes issus de l'immigration (Kao \& Tienda, 1995, 1998; Vallet, 2005; Brinbaum \& Cebolla-Boado, 2007; Van de Werfhorst \& Van Tubergen, 2007; Becker \& Schubert, 2011). Ils pointent par ailleurs du doigt l'insuffisance des renseignements dont disposent les jeunes au sujet des alternatives aux études supérieures (Relikowski et al., 2012), notamment des filières duales existant en Suisse et en Allemagne, les discriminations dont ils pourraient faire l'objet dans leur recherche d'une place d'apprentissage (Kristen et al., 2008), ainsi que les chances plus grandes de faire valoir à l'étranger un diplôme d'une haute école plutôt qu'un certificat sanctionnant la réussite d'une formation professionnelle, dans l'hypothèse d'un retour ultérieur au pays des jeunes issus de l'immigration (Jonsson \& Rudolphi, 2010).

Compte tenu des meilleures perspectives qui s'offrent aux femmes sur le marché du travail (Becker \& Müller, 2011) combinées à l'évolution des mentalités quant à la répartition des tâches entre hommes et femmes, la participation à la formation des femmes a augmenté, si bien que celles-ci sont désormais plus nombreuses que leurs homologues masculins à terminer des études secondaires (Buchmann et al., 2008). Malgré cette tendance, en Suisse et en Allemagne, 
le taux de femmes à poursuivre leurs études au-delà du degré secondaire alors qu'elles ont les qualifications requises, reste moins élevé que celui des hommes. Pour expliquer ce phénomène pour l'Allemagne, Lörz \& Schindler (2011) renvoient à la perception qu'ont les femmes d'avoir moins de chance que leurs études soient couronnées de succès et à leur plus grande sensibilité aux coûts. Enfin, les objectifs que se fixent hommes et femmes dans la vie divergent: alors que les premiers sont soucieux d'engranger un bon salaire et de jouir de prestige social, les secondes accordent plus d'importance à l'engagement social.

Si l'on entend, pour le groupe des migrants issus de milieux sociaux défavorisés qui nous intéressent ici, déduire des hypothèses sur le recoupement entre inégalités dues au sexe et au contexte migratoire et la poursuite d'études supérieures, il faut invoquer deux facteurs d'explication: d'une part les chances d'accès au marché du travail et le rendement de l'éducation dans le pays d'accueil, de l'autre les stéréotypes de genre véhiculés dans les pays d'origine. S'agissant du premier facteur, il convient tout d'abord de s'interroger sur la mesure dans laquelle ces chances sont différentes pour les candidats nationaux et pour ceux qui sont issus de l'immigration. A cet égard, le handicap que rencontrent les jeunes hommes issus de l'immigration appartenant à des classes sociales défavorisées dans les systèmes éducatifs suisse et allemand caractérisés par les qualifications pour le passage important dans la formation professionnelle (en entreprise) a été prouvé (voir pour la Suisse Fibbi et al., 2006; Imdorf, 2010; pour l'Allemagne: Diehl et al., 2009). Pour expliquer les inégalités observées au niveau de ce passage entre la population suisse et la population immigrée, les approches discriminatoires notamment semblent pertinentes. L'approche de la "discrimination statistique» reposant sur Arrow (1973) et Phelps (1972) désigne le manque d'information comme origine de la différence de traitement appliqué aux membres de différents groupes sociaux. Ainsi, suivant ce raisonnement, les employeurs n'étant pas au fait de la productivité réelle des candidats, ils se réferent aux valeurs moyennes du groupe social dont ils sont issus. L'hypothèse d'une «préférence pour la discrimination» basée sur les considérations de Gary S. Becker (1957) impute ces processus aux inclinations individuelles ainsi qu'aux préjugés des acteurs économiques, des employeurs par exemple. Le constat établi par Imdorf (2010), selon lequel les employeurs suisses (en particulier dans les PME) prêtent aux hommes issus de l'immigration appartenant à des classes sociales défavorisées un potentiel de nuisance plus élevé, concorde avec ces approches.

Sur la base de longues recherches, on peut avancer qu'en comparaison avec la population de référence, les jeunes issus de l'immigration tablent sur le fait qu'une formation autre que professionnelle entraînera des coûts élevés, compte tenu de l'éventualité de faire l'objet de discrimination dans la recherche d'une place d'apprentissage conjuguée à l'hypothèse fondée sur la théorie de l'anticipation de valeur. À contrario, il devrait en résulter un effet positif sur la probabilité que ces jeunes issus de l'immigration appartenant à des classes sociales défavorisées, toutes choses égales par ailleurs, optent pour des études supérieures 
dans des systèmes éducatifs dans lesquels la formation en entreprise revêt une grande importance.

Dans la perspective des stéréotypes de genre plutôt traditionnels dans certains pays, il est intéressant de se demander à quel point ils sont reproduits dans les sociétés d'accueil européennes. Si l'on part du principe que les groupes de migrants perpétuent ces représentations et, partant, que ces familles investissent plutôt sur les hommes sur le plan de la formation, on devrait observer, tant en Suisse, qu'en Allemagne et en France, que les hommes sont privilégiés aux dépens des femmes. Toutefois, on ne peut exclure que ces influences aient eu un impact déjà plus tôt dans le parcours éducatif. D'un point de vue empirique, il s'agit en outre de tenir compte du constat tiré par Fleischmann \& Kristen (2011), selon lequel la participation à la formation des femmes issues de l'immigration s'inspire davantage des modèles du pays d'accueil que du pays d'origine.

\section{Stratégie d'analyse}

Pour identifier dans une première étape si, pour les femmes et les hommes, des groupes de migrants sont favorisés ou désavantagés dans le passage aux études supérieures par rapport aux femmes et aux hommes locaux - et si oui, lesquels - sont d'abord contrôlés uniquement les types de contextes migratoires (voir plus bas), séparément pour les femmes et les hommes (modèle 1). Les inégalités liées au contexte migratoire étant, comme nous l'avons mis en évidence plus haut, imputables en particulier aux inégalités sociales, nous analyserons dans une deuxième étape, toujours pour les femmes et les hommes séparément, dans quelle mesure les inégalités dues au contexte migratoire peuvent être expliquées par l'origine sociale et les résultats scolaires (modèle 2). À ce stade, on tentera également de déterminer si - indépendamment de l'origine sociale et des résultats scolaires - l'hypothèse selon laquelle les jeunes migrants issus de classes sociales défavorisées affichent un taux de passage plus élevé dans l'enseignement supérieur se vérifie autant pour les deux sexes, ou si, en Suisse et en Allemagne, les jeunes hommes appartenant à ces milieux sont effectivement plus nombreux à entamer des études supérieures, compte tenu de la crainte d'être victimes de discrimination dans la recherche d'une place d'apprentissage. Dans une dernière étape, on s'intéressera aux effets d'interaction, soit aux intersections à l'incidence multiplicative, entre le contexte migratoire et le sexe (modèle 3).

\section{Données et méthodes}

Pour analyser la thématique du passage du système éducatif au marché de l'emploi en Suisse, on a utilisé les données relevées dans le cadre du projet TREE, Transitions de l'École à l'Emploi, (TREE, 2010) Cette étude longitudinale, fondée 
sur l'enquête PISA de 2000 portant sur un échantillon de quelque 6000 jeunes, s'est déclinée en huit épisodes organisés entre 2001 et 2010. Pour la France, le Panel d'élèves du second degré, recrutement 1995, offrait également des données longitudinales adaptées, celle-ci reposant sur un premier sondage réalisé auprès d'environ 11000 jeunes âgés de 12 ans en 1995. Quant à l'Allemagne, la consultation - en 2002 et en 2005 - de quelque 8000 étudiants ayant décroché en 2002 dans ce pays le sésame leur permettant d'accéder à des études supérieures, fournissait elle aussi des données propres à analyser le phénomène du passage dans les hautes écoles.

Pour ces trois pays, seuls les élèves pouvant prétendre aux études supérieures ont été pris en considération. ${ }^{1}$ Par ailleurs, les jeunes issus de l'immigration n'ont été retenus que s'ils pouvaient être facilement classés par origine géographique, les jeunes appartenant simultanément à plusieurs groupes de migrants (p. ex. pour la Suisse, les jeunes dont le père est né en Suisse et la mère en Turquie) ayant été exclus.

Pour procéder à l'analyse, des modèles de régression logistique binaire ont été estimés. Sont présentés les average marginal effects (AME), afin de pouvoir comparer entre eux les différents modèles par sexe et pays d'accueil. En effet, ces coefficients sont robustes vis-à-vis de l'hétérogénéité non observée entre les divers échantillons partiels (Wooldridge, 2002: p. 470). Les effets marginaux moyens se rapportent à l'effet moyen de chacune des variables indépendantes sur la probabilité d'entreprendre des études supérieures dans les trois ans suivant l'obtention du diplôme ad hoc (catégorie 1), lorsque toutes les autres variables indépendantes du modèle affichent leur valeur moyenne respective. ${ }^{2}$ Quant à la catégorie de référence de la variable dépendante (catégorie 0 ), elle correspond aux personnes pouvant prétendre à des études supérieures ayant choisi d'y renoncer.

A toujours été codé en tant que variable indépendante l'existence d'une forme de contexte migratoire, lorsque la personne interrogée elle-même, l'un de ses parents ou ses deux parents sont nés à l'étranger (pour un débat au sujet des différentes méthodes, voir Gresch \& Kristen 2011). S'agissant du groupe de jeunes issus de la migration appartenant à un milieu social défavorisé sur lequel la présente analyse met l'accent, cela concerne, en Suisse, en premier lieu les jeunes de la première vague migratoire datant des années 1940 (essentiellement des travailleurs immigrés espagnols et italiens) et de la deuxième vague migratoire datant des années 1980 (travailleurs immigrés de l'ex-Yougoslavie, du Portugal et les réfugiés en provenance de Turquie, d'ex-Yougoslavie et d'Albanie). ${ }^{3}$ Même si ce n'est pas sur eux que les projecteurs sont braqués dans la présente contribution en raison de leur origine privilégiée, les jeunes issus de l'immigration allemande, autrichienne, française et belge, de même que d' «autres pays» ont été pris en compte, puisqu'ils permettent, par contraste, de mettre en évidence la situation des immigrés défavorisés. En France, ce sont en particulier les jeunes issus de l'immigration maghrébine (Algériens, Marocains et Tunisiens) qui présentent le handicap social le plus marqué par rapport aux jeunes Français. À ceux-ci 
viennent par ailleurs s'ajouter les jeunes originaires de l'Europe du Sud. Les autres jeunes issus de l'immigration ont été rangés dans le groupe de contrôle, celui des «autres» contextes migratoires (entre autres des pays européens industrialisés). En Allemagne, entrent dans le groupe des jeunes socialement les moins favorisés les immigrés en provenance de Turquie, d'Europe du Sud et d'Europe de l'Est (ex-Yougoslavie comprise). Contrairement à la Suisse et à la France, pour le cas allemand, le contexte migratoire a été opérationnalisé au moyen de la nationalité des jeunes pouvant prétendre à des études supérieures. En introduisant une variable muette pour la première génération de migrants - c'està-dire pour les diplômés ayant eux-mêmes vécu des expériences migratoires -, les résultats de chacun des groupes de migrants se rapportent aux migrants de la deuxième génération, soit ceux dont les parents ont immigré, mais qui, eux, sont nés dans le pays d'accueil et y ont effectué l'ensemble de leur parcours éducatif.

Afin d'opérationnaliser l'origine sociale, on s'est fondé pour la France et l'Allemagne, sur le niveau de formation le plus élevé atteint par l'un des parents (variable muette: 1 - études secondaires de niveau CITE 5A versus 0 - formation moins élevée), et pour la Suisse, sur l'indice métrique du statut socio-économique (HISEI), basé sur des données internationales relatives au revenu et au niveau de formation (Ganzeboom et al., 1992). Pour les trois pays, le sexe est saisi de manière dichotomique, la catégorie 1 correspondant aux hommes, la catégorie de référence, 0 , aux femmes.

Afin de pouvoir tenir compte des effets primaires des origines sociale et migratoire au titre de prestations différentielles, ont été contrôlées dans le cadre de l'analyse les notes de fin d'études ou celles sanctionnant le diplôme donnant accès aux hautes écoles, ces notes ayant été transformées en variables muettes (quatre catégories: note tombant dans le tercile inférieur, note tombant dans le tercile moyen, note tombant dans le tercile supérieur et note manquante $)^{4}$. Le système de notation varie dans ces trois pays: en Suisse, la meilleure note est 6 (tercile inférieur: de 4 à 4,55, tercile moyen: de 4,6 à 4,95, tercile supérieur: de 5 à 6), en France, c'est 20 (tercile inférieur: de 10 à 10,6, tercile moyen: de 10,61 à 11,98 , tercile supérieur: de 12 à 20), tandis qu'en Allemagne, c'est 1 (tercile inférieur: de 4 à 2,8, tercile moyen: de 2,7 à 2,1, tercile supérieur: de 2 à 1 ).

Les distributions marginales des variables des modèles dans la perspective des trois échantillons sont représentées dans le tableau $\mathrm{n}^{\circ} 1$.

\section{Résultats}

Les résultats des échantillons suisses (données TREE) se trouvent dans le tableau $\mathrm{n}^{\circ} 2$. Alors que chez les hommes, dans le modèle 1 , certains groupes de migrants se distinguent sensiblement des suisses pour le passage dans les hautes écoles, on ne constate pas chez les femmes d'influence significative du contexte migratoire sur la chance d'entreprendre ou non des études supérieures. 
Tableau 1: Répartition des variables du modèle

\begin{tabular}{|l|c|c|c|c|c|c|}
\hline Suisse: TREE* & Total & $\begin{array}{c}\text { Sans } \\
\text { histoire } \\
\text { migratoire } \\
\text { (Réf.) }\end{array}$ & $\begin{array}{c}\text { Ex-You- } \\
\text { goslavie, } \\
\text { Albanie/ } \\
\text { Kosovo, } \\
\text { Turquie, } \\
\text { Portugal }\end{array}$ & $\begin{array}{c}\text { Espagne, } \\
\text { Italie }\end{array}$ & $\begin{array}{c}\text { Allemagne, } \\
\text { Autriche, } \\
\text { France, } \\
\text { Belgique }\end{array}$ & Autres \\
\hline $\begin{array}{l}\text { Entrée dans une haute } \\
\text { école (\%) }\end{array}$ & 65,4 & 64,3 & 52,3 & 65,3 & 64,9 & 64,5 \\
\hline $1^{\text {e génération (\%) }}$ & 9,7 & - & 56,1 & 9,9 & 8,9 & 6,2 \\
\hline Note 1 ${ }^{\text {er tercile }}$ & 19,5 & 22,8 & 14,9 & 20,3 & 20,0 & 20,6 \\
\hline Note 2e tercile & 21,2 & 22,1 & 10,1 & 21,6 & 20,5 & 21,5 \\
\hline Note 3e tercile & 21,8 & 20,6 & 24,3 & 21,3 & 21,7 & 21,1 \\
\hline Note manquante & 37,5 & 34,6 & 50,6 & 36,9 & 37,8 & 36,9 \\
\hline Maturité & 54,5 & 52,8 & 32,0 & 54,1 & 53,9 & 52,5 \\
\hline HISEI (moy.) & 55,7 & 56,2 & 39,0 & 49,9 & 59,4 & 62,2 \\
\hline N (non pondéré) & 2.537 & 1.637 & 143 & 218 & 174 & 273 \\
\hline
\end{tabular}

\begin{tabular}{|l|c|c|c|c|c|c|c|}
\hline Allemagne: HIS & Total & $\begin{array}{c}\text { Non-na- } \\
\text { tionaux } \\
\text { (Réf.) }\end{array}$ & Turquie & $\begin{array}{c}\text { Europe } \\
\text { du Sud }\end{array}$ & $\begin{array}{c}\text { Europe } \\
\text { de } \\
\text { ''Ouest }\end{array}$ & $\begin{array}{c}\text { Europe } \\
\text { de l'Est, } \\
\text { Ex-You- } \\
\text { goslavie }\end{array}$ & Autres \\
\hline $\begin{array}{l}\text { Entrée dans une haute } \\
\text { école (\%) }\end{array}$ & 72,9 & 72,9 & 71,4 & 60,6 & 81,8 & 78,7 & 72,7 \\
\hline $1^{\text {e génération (\%) }}$ & 4,9 & - & 7,1 & 33,3 & 24,2 & 53,2 & 63,6 \\
\hline Note 1 ${ }^{\text {er tercile }}$ & 28,0 & 28,6 & 5,8 & 26,0 & 38,3 & 14,0 & 21,1 \\
\hline Note 2e tercile & 37,7 & 38,4 & 24,4 & 24,7 & 26,7 & 25,0 & 34,2 \\
\hline Note 3e tercile & 38,9 & 37,6 & 27,1 & 52,1 & 38,3 & 68,0 & 47,4 \\
\hline Note manquante & 0,9 & 1,0 & 1,2 & 1,4 & 0,0 & 0,0 & 0,0 \\
\hline Abitur & 76,4 & 77,1 & 34,9 & 52,1 & 85,0 & 65,0 & 60,5 \\
\hline $\begin{array}{l}\text { Formation la plus élevée } \\
\text { achevée par les parents: } \\
\text { diplôme supérieur (\%) }\end{array}$ & 44,8 & 45,4 & 11,6 & 31,5 & 75,0 & 33,0 & 47,4 \\
\hline N (non pondéré) & 7.175 & 7.023 & 28 & 33 & 33 & 47 & 11 \\
\hline
\end{tabular}




\begin{tabular}{|l|c|c|c|c|c|}
\hline \multicolumn{1}{|l|}{ France: Panel d'élèves 1995 } & Total & $\begin{array}{c}\text { Sans histoire } \\
\text { migratoire } \\
\text { (Réf.) }\end{array}$ & Maghreb & Europe du Sud & Autres \\
\hline $\begin{array}{l}\text { Entrée dans une haute } \\
\text { école (\%) }\end{array}$ & 65,7 & 65,6 & 68,6 & 56,2 & 70,6 \\
\hline $1^{\text {e génération (\%) }}$ & 4,4 & - & 31,2 & 27,0 & 20,0 \\
\hline Note 1 ${ }^{\text {er }}$ tercile & 31,02 & 32,13 & 22,51 & 28,57 & 27,73 \\
\hline Note 2e tercile & 30,29 & 30,06 & 31,55 & 29,48 & 31,76 \\
\hline Note 3e tercile & 30,29 & 29,27 & 36,66 & 31,91 & 31,60 \\
\hline Note manquante & 4,84 & 5,09 & 4,99 & 4,26 & 3,53 \\
\hline Baccalauréat général & 31,21 & 55,64 & 48,84 & 47,72 & 58,66 \\
\hline $\begin{array}{l}\text { Formation la plus élevée } \\
\text { achevée par les parents: } \\
\text { diplôme supérieur (\%) }\end{array}$ & 30,7 & 32,6 & 18,3 & 12,7 & 32,3 \\
\hline N (non pondéré) & 9.192 & 7.728 & 921 & 308 & 335 \\
\hline
\end{tabular}

* Conformément aux recommandations de Sacchi (2011), les analyses portant sur la Suisse ont fait l'objet d'une pondération longitudinale.

Ainsi, les hommes de la deuxième génération de migrants originaires d'ex-Yougoslavie, d'Albanie, de Turquie ou du Portugal affichent-ils une probabilité supérieure de 20,7 points aux hommes suisses de poursuivre leurs études dans le degré tertiaire.

Dans le modèle 2, on observe que l'origine sociale (statut professionnel le plus élevé des parents) a un impact positif sur la probabilité de passer cette étape, pour les hommes comme pour les femmes. La note obtenue en fin de parcours secondaire joue un rôle plus important chez les hommes (une note située dans les terciles moyen et supérieur augmentant la probabilité de franchir le pas) que chez les femmes, où seules les étudiantes tombant dans le tercile moyen se démarquent manifestement - à hauteur de 10 points - des jeunes filles suisses.

Le modèle 3, qui a été constitué à partir de l'ensemble de l'échantillon suisse - hommes et femmes confondus -, suggère que, indépendamment des termes d'interaction résultant du sexe et des différents contextes migratoires, le sexe n'a pas d'effet significatif. S'agissant desdits termes d'interaction, celui conjuguant le sexe masculin aux régions d'origine «ex-Yougoslavie, Albanie, Turquie, Portugal» s'avère déterminant. Ainsi, la probabilité des hommes de la deuxième génération de migrants provenant de cette région pouvant prétendre aux études supérieures à effectivement faire ce choix dépasse de 24 points celle des femmes. Ce constat est en droite ligne avec les hypothèses de départ, à savoir que dans ce groupe de migrants, les stéréotypes de genre sont plus persistants et que les formations professionnelles sont évitées 5 . 
Tableau 2: Suisse: Chances d'accès à la formation selon le sexe, le contexte migratoire et l'origine

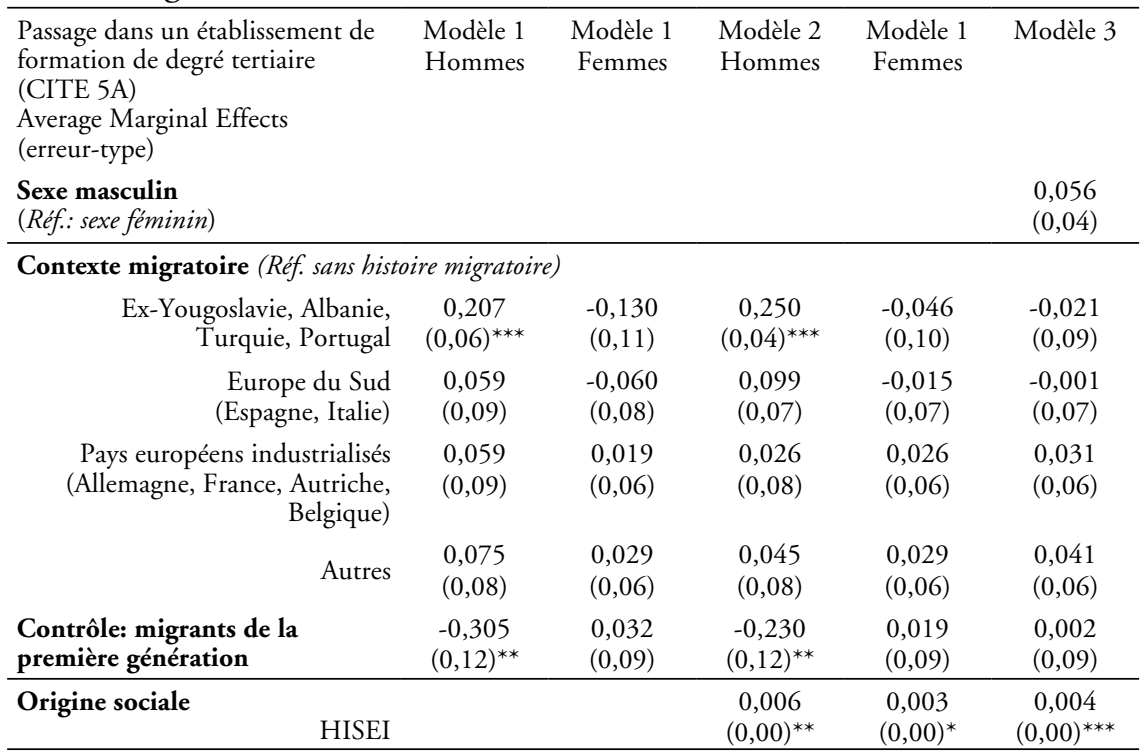

Note au diplôme donnant accès à l'enseignement supérieur (Réf. tercile inférieur)

$\begin{array}{cccc}\text { Tercile moyen } & 0,123 & 0,105 & 0,115 \\ \text { Tercile supérieur } & (0,06)^{*} & (0,06) \dagger & (0,04)^{* *} \\ & 0,154 & 0,084 & 0,118 \\ \text { Note manquante } & (0,06)^{*} & (0,06) & (0,04)^{* *} \\ & -0,025 & 0,011 & -0,008 \\ & (0,07) & (0,06) & (0,05)\end{array}$

Effets d'interactions sexe $\mathbf{x}$ contexte migratoire Sexe masculin $\mathrm{x}$ (Réf. nationaux)

Ex-Yougoslavie, Albanie, Turquie, Portugal

Europe du Sud

(Espagne, Italie)

Pays européens industrialisés

(Allemagne, France, Autriche,

Belgique)

Autres
0,240 $(00,04)^{* * *}$

0,111

$(0,07)$

$-0,016$

$(0,11)$

$-0,014$

$(0,11)$

$-0,320$

$(0,16)^{*}$

$-2,190$

\begin{tabular}{lccccc}
\hline Constante & $-0,495$ & $-0,718$ & $-2,207$ & $-1,871$ & $-2,190$ \\
$\mathrm{~N}$ & 886 & 1.342 & 886 & 1.342 & 2.228 \\
$\mathrm{R}^{2}$ de Nagelkerke & 0,394 & 0,302 & 0,448 & 0,311 & 0,325 \\
\hline
\end{tabular}

* Données TREE-Daten, pondérées, calculs propres; variables de contrôle supplémentaires dans tous les modèles: régions linguistiques suisses (Suisse alémanique, Suisse romande et Tessin), type du diplôme donnant accès à l'enseignement supérieur (Maturité vs. diplôme donnant un accès restreint à l'enseignement supérieur) 
En Allemagne comme en Suisse, dans le modèle 1, les migrants de la deuxième génération ne semblent pas être notablement désavantagés (tableau $\mathrm{n}^{\circ} 3$ ). À l'inverse même, les hommes en provenance d'Europe de l'Est et d'ex-Yougoslavie présentent même une probabilité supérieure de 15,6 points par rapport aux étudiants allemands de s'engager sur la voie des études de degré tertiaire. De même, les jeunes femmes issues de pays industrialisés d'Europe de l'Ouest optent plus souvent que les jeunes Allemandes pour les études supérieures.

Ici encore, l'origine sociale et la note de fins d'études ont un impact significatif sur le choix de formation. En outre, indépendamment de ces deux facteurs, la probabilité de s'immatriculer dans une haute école est supérieure de 15 points pour les hommes et les femmes venues d'Europe de l'Est (ex-Yougoslavie comprise) en comparaison avec les Allemands. Certes, pour les hommes, cela confirme l'hypothèse de départ. En revanche, pour les femmes issues de ce groupe de migrants, le taux de passage dans le supérieur étant également plus élevé, qui plus est dans les mêmes proportions, cela tend à prouver que la discrimination escomptée formulée plus haut ne suffit pas à expliquer les choix de formation.

Dans le modèle 3, on constate que les données allemandes confirment que les hommes sont plus nombreux à poursuivre leurs études au degré tertiaire que les femmes. Par contre, nul recoupement entre sexe et contexte migratoire ne se dessine, dans le sens d'un renforcement ou d'interactions. Si l'on prend en considération le caractère cumulatif des inégalités, on obtient un taux de passage particulièrement élevé pour les hommes appartenant à la deuxième génération de migrants en provenance d'Europe de l'Est.

S'agissant du modèle 1, la France se distingue de ses voisins allemand et suisse (tableau $n^{\circ} 4$ ). Alors que dans ces deux pays, les hommes affichent un taux de passage dans le degré tertiaire supérieur aux étudiants nationaux, au moins dans l'un des groupes de migrants défavorisés, cette tendance ne se vérifie ici que pour les sujets de sexe féminin. Ainsi, la probabilité que les femmes de la deuxième génération issue de l'immigration maghrébine pouvant prétendre à des études supérieures s'engagent dans cette voie dépasse de 13,8 points celle des Françaises.

Le modèle 2 montre que, comme en Suisse, l'origine sociale est déterminante pour la poursuite des études, davantage chez les femmes que chez les hommes d'ailleurs. On observe de surcroît que celle-ci est d'autant plus grande que les notes obtenues au baccalauréat tombent dans les terciles moyen ou supérieur. $\mathrm{Si}$, contrairement au modèle 1 , on retrouve cette probabilité plus élevée chez les hommes de la deuxième génération issue de l'immigration maghrébine dans le modèle 2, en termes relatifs, l'effet est mineur et peu significatif. Par contre, les femmes de la même origine affichent, indépendamment de leur milieu social et de leurs résultats scolaires, une probabilité supérieure de 15,4 points que les jeunes Françaises à opter pour cette voie. 
Tableau 3: Allemagne: Chances d'accès à la formation selon le sexe, le contexte migratoire et l'origine

\begin{tabular}{|c|c|c|c|c|c|}
\hline $\begin{array}{l}\text { Passage dans un établissement de } \\
\text { formation de degré tertiaire } \\
\text { (CITE 5A) } \\
\text { Average Marginal Effects } \\
\text { (erreur-type) }\end{array}$ & $\begin{array}{l}\text { Modèle } 1 \\
\text { Hommes }\end{array}$ & $\begin{array}{l}\text { Modèle } 1 \\
\text { Femmes }\end{array}$ & $\begin{array}{l}\text { Modèle } 2 \\
\text { Hommes }\end{array}$ & $\begin{array}{l}\text { Modèle } 1 \\
\text { Femmes }\end{array}$ & Modèle 3 \\
\hline $\begin{array}{l}\text { Sexe masculin } \\
\text { (Réf. sexe féminin) }\end{array}$ & & & & & $\begin{array}{c}0,088 \\
(0,01)^{* * *}\end{array}$ \\
\hline \multicolumn{6}{|l|}{ Contexte migratoire (Réf. nationaux) } \\
\hline Europe du Sud & $\begin{array}{r}-0,109 \\
(0,13)\end{array}$ & $\begin{array}{c}-0,008 \\
(0,10)\end{array}$ & $\begin{array}{c}-0,096 \\
(0,13)\end{array}$ & $\begin{array}{l}0,008 \\
(0,10)\end{array}$ & $\begin{array}{l}-0,001 \\
(0,09)\end{array}$ \\
\hline Turquie & $\begin{array}{l}0,050 \\
(0,09)\end{array}$ & $\begin{array}{l}0,037 \\
(0,11)\end{array}$ & $\begin{array}{l}0,100 \\
(0,06)\end{array}$ & $\begin{array}{l}0,115 \\
(0,08)\end{array}$ & $\begin{array}{l}0,091 \\
(0,07)\end{array}$ \\
\hline Europe de l'Ouest & $\begin{array}{l}0,011 \\
(0,11)\end{array}$ & $\begin{array}{c}0,137 \\
(0,08) \dagger\end{array}$ & $\begin{array}{c}-0,062 \\
(0,13)\end{array}$ & $\begin{array}{l}0,111 \\
(0,09)\end{array}$ & $\begin{array}{l}0,105 \\
(0,08)\end{array}$ \\
\hline Europe de l'Est, Ex-Yougoslavie & $\begin{array}{c}0,156 \\
(0,06)^{*}\end{array}$ & $\begin{array}{l}0,090 \\
(0,07)\end{array}$ & $\begin{array}{c}0,146 \\
(0,06)^{*}\end{array}$ & $\begin{array}{c}0,147 \\
(0,05)^{* *}\end{array}$ & $\begin{array}{c}0,130 \\
(0,04)^{* *}\end{array}$ \\
\hline Autres & $\begin{array}{l}0,053 \\
(0,16)\end{array}$ & $\begin{array}{l}0,084 \\
(0,15)\end{array}$ & $\begin{array}{l}0,036 \\
(0,16)\end{array}$ & $\begin{array}{l}0,070 \\
(0,14)\end{array}$ & $\begin{array}{l}0,037 \\
(0,15)\end{array}$ \\
\hline $\begin{array}{l}\text { Contrôle: migrants de la première } \\
\text { génération }\end{array}$ & $\begin{array}{r}-0,025 \\
(0,04) \\
\end{array}$ & $\begin{array}{l}-0,061 \\
(0,03) \dagger\end{array}$ & $\begin{array}{r}-0,002 \\
(0,04) \\
\end{array}$ & $\begin{array}{r}-0,037 \\
(0,03) \\
\end{array}$ & $\begin{array}{r}-0,030 \\
(0,02) \\
\end{array}$ \\
\hline \multicolumn{6}{|c|}{ Origine sociale Niveau de formation le plus élevé des parents } \\
\hline Formation supérieure (CITE 5A) & & & $\begin{array}{c}0,102 \\
(0,02)^{* * *}\end{array}$ & $\begin{array}{c}0,146 \\
(0,01)^{* * *}\end{array}$ & $\begin{array}{c}0,129 \\
(0,01)^{* * *}\end{array}$ \\
\hline
\end{tabular}

Note au diplôme donnant accès à l'enseignement supérieur (Réf. tercile inférieur)

$\begin{array}{cccc}\text { Tercile moyen } & 0,101 & 0,122 & 0,115 \\ & (0,02)^{* * *} & (0,02)^{* * *} & (0,01)^{* * *} \\ \text { Tercile supérieur } & 0,175 & 0,235 & 0,213 \\ & (0,02)^{* * *} & (0,01)^{* * *} & (0,01)^{* * *} \\ \text { Note manquante } & -0,070 & 0,003 & -0,019 \\ & (0,10) & (0,07) & (0,06)\end{array}$

Effets d'interactions sexe $\mathbf{x}$ contexte migratoire. Sexe masculin $\mathrm{x}$ (Réf. nationaux)

$$
\text { Europe du Sud }
$$

Turquie

Europe de l'Ouest

Europe de l'Est, Ex-Yougoslavie

Autres

Sexe masculin $\mathrm{x}$

migrants de la première génération

\begin{tabular}{lccccc} 
migrants de la première génération & \multicolumn{3}{c}{$(0,28)$} \\
\hline Constante & 0,858 & $-0,193$ & 0,163 & $-0,959$ & $-0,699$ \\
$\mathrm{~N}$ & 2.478 & 4.655 & 2.478 & 4.655 & 7.133 \\
$\mathrm{R}^{2}$ de Nagelkerke & 0,02 & 0,08 & 0,10 & 0,19 & 0,16 \\
\hline
\end{tabular}

Source: HIS 2002, calculs propres; variables de contrôle supplémentaires dans tous les modèles: type de diplôme de fin d'études secondaires (Abitur vs. diplôme donnant un accès restreint à l'enseignement supérieur) 
Tableau 4: France: Chances d'accès à la formation selon le sexe, le contexte migratoire et l'origine

\begin{tabular}{|c|c|c|c|c|c|}
\hline $\begin{array}{l}\text { Passage dans un établissement de } \\
\text { formation de degré tertiaire } \\
\text { (CITE 5A) } \\
\text { Average Marginal Effects } \\
\text { (erreur-type) }\end{array}$ & $\begin{array}{l}\text { Modèle } 1 \\
\text { Hommes }\end{array}$ & $\begin{array}{l}\text { Modèle } 1 \\
\text { Femmes }\end{array}$ & $\begin{array}{l}\text { Modèle } 2 \\
\text { Hommes }\end{array}$ & $\begin{array}{l}\text { Modèle } 2 \\
\text { Femmes }\end{array}$ & Modèle 3 \\
\hline $\begin{array}{l}\text { Sexe masculin } \\
\text { (Réf. sexe féminin) }\end{array}$ & & & & & $\begin{array}{c}-0,020 \\
(0,02) \\
\end{array}$ \\
\hline \multicolumn{6}{|c|}{ Contexte migratoire (Réf. sans histoire migratoire) } \\
\hline $\begin{array}{l}\text { Maghreb (Algérie, } \\
\text { Maroc, Tunisie) }\end{array}$ & $\begin{array}{l}0,062 \\
(0,04)\end{array}$ & $\begin{array}{c}0,138 \\
(0,02)^{* * *}\end{array}$ & $\begin{array}{c}0,072 \\
(0,04) \dagger\end{array}$ & $\begin{array}{c}0,154 \\
(0,02)^{* * *}\end{array}$ & $\begin{array}{c}0,168 \\
(0,07)^{* * *}\end{array}$ \\
\hline Europe du Sud & $\begin{array}{l}0,003 \\
(0,06)\end{array}$ & $\begin{array}{c}-0,041 \\
(0,05)\end{array}$ & $\begin{array}{l}0,020 \\
(0,06)\end{array}$ & $\begin{array}{c}-0,044 \\
(0,05)\end{array}$ & $\begin{array}{r}-0,051 \\
(0,06)\end{array}$ \\
\hline Autres & $\begin{array}{l}0,042 \\
(0,06)\end{array}$ & $\begin{array}{c}0,090 \\
(0,04)^{*}\end{array}$ & $\begin{array}{l}0,050 \\
(0,06)\end{array}$ & $\begin{array}{c}0,092 \\
(0,03)^{*}\end{array}$ & $\begin{array}{c}0,101 \\
(0,04)^{*}\end{array}$ \\
\hline $\begin{array}{l}\text { Contrôle: migrants de la } \\
\text { première génération }\end{array}$ & $\begin{array}{r}-0,005 \\
(0,06) \\
\end{array}$ & $\begin{array}{c}-0,044 \\
(0,05) \\
\end{array}$ & $\begin{array}{l}0,029 \\
(0,06) \\
\end{array}$ & $\begin{array}{r}-0,017 \\
(0,04) \\
\end{array}$ & $\begin{array}{l}-0,029 \\
(0,05)^{*}\end{array}$ \\
\hline \multicolumn{6}{|l|}{ Origine sociale } \\
\hline $\begin{array}{l}\text { Niveau de formation le plus élevé } \\
\text { des parents } \\
\text { Formation supérieure (CITE 5A) }\end{array}$ & & & $\begin{array}{c}0,044 \\
(0,02) \dagger\end{array}$ & $\begin{array}{c}0,095 \\
(0,02)^{* * *}\end{array}$ & $\begin{array}{c}0,071 \\
(0,02)^{* * *}\end{array}$ \\
\hline
\end{tabular}

\begin{tabular}{|c|c|c|c|c|c|}
\hline \multicolumn{6}{|c|}{ Note au diplôme donnant accès à l'enseignement supérieur (Réf. tercile inférieur) } \\
\hline \multicolumn{3}{|l|}{ Tercile moyen } & $\begin{array}{c}0,073 \\
(0,03)^{* *}\end{array}$ & $\begin{array}{c}0,051 \\
(0,02)^{*}\end{array}$ & $\begin{array}{c}0,060 \\
(0,02)^{* * *}\end{array}$ \\
\hline \multicolumn{3}{|l|}{ Tercile supérieur } & $\begin{array}{c}0,153 \\
(0,03)^{* * *}\end{array}$ & $\begin{array}{c}0,151 \\
(0,02)^{* * *}\end{array}$ & $\begin{array}{c}0,156 \\
(0,02)^{* * *}\end{array}$ \\
\hline \multicolumn{3}{|l|}{ Note manquante } & $\begin{array}{l}0,069 \\
(0,05)\end{array}$ & $\begin{array}{c}0,126 \\
(0,02)^{* * *}\end{array}$ & $\begin{array}{c}0,102 \\
(0,03)^{* * *}\end{array}$ \\
\hline \multicolumn{6}{|c|}{ Effets d'interactions sexe $\mathbf{x}$ contexte migratoire Sexe masculin $\mathrm{x}$ (Réf. nationaux) } \\
\hline \multicolumn{2}{|c|}{$\begin{array}{l}\text { Maghreb (Algérie, } \\
\text { Maroc, Tunisie) }\end{array}$} & & & & $\begin{array}{l}-0,142 \\
(0,07)^{*}\end{array}$ \\
\hline \multicolumn{2}{|l|}{ Europe du Sud } & & & & $\begin{array}{l}0,069 \\
(0,07)\end{array}$ \\
\hline \multicolumn{2}{|l|}{ Autres } & & & & $\begin{array}{r}-0,069 \\
(0,09)\end{array}$ \\
\hline $\begin{array}{l}\text { Sexe masculin } \mathrm{x} \\
\text { migrants de la première } \\
\text { génération }\end{array}$ & & & & & $\begin{array}{l}0,065 \\
(0,07)\end{array}$ \\
\hline Constante & $-0,697$ & $-0,760$ & -1.130 & -1.303 & $-0,117$ \\
\hline $\mathrm{N}$ & 2351 & 2870 & 2351 & 2870 & 5221 \\
\hline $\mathrm{R}^{2}$ de Nagelkerke & 0,23 & 0,25 & 0,25 & 0,27 & 0,29 \\
\hline
\end{tabular}

Source: Panel d'élève 1995, calculs propres; variables de contrôle supplémentaires dans tous les modèles: type de diplôme de fin d'études secondaires (Baccalauréat général vs. Baccalauréat technique/autres).

(Traduction: Caroline Suter-Sturm) 
Le modèle 3 ne révèle pas de différences entre les sexes en matière d'accès aux hautes écoles. En revanche, il y a bien ici des recoupements entre sexe et contexte migratoire, dans le sens d'un renforcement ou d'interactions, puisque l'avantage des hommes issus de l'immigration maghrébine en termes de passage dans le degré tertiaire est moins grand que celui des femmes de la même origine.

\section{Synthèse et conclusions}

Si l'on s'intéresse aux hypothèses de départ, à savoir qu'il existerait des recoupements entre les deux axes d'inégalité que sont le sexe et le contexte migratoire, elles se sont vérifiées pour la Suisse et l'Allemagne, puisque le taux de passage dans le supérieur des hommes issus de l'immigration appartenant à des milieux défavorisés (de la deuxième génération) est effectivement plus élevé. On ne peut cependant pas exclure que ces effets, pour le moins en Suisse, soient imputables non seulement à la répartition traditionnelle des rôles, mais également à l'évaluation que ces groupes de migrants font des alternatives qui s'offrent à eux en termes de formation, puisque ces taux de passage sont également plus élevés que ceux de leurs homologues féminins.

$\mathrm{Ni}$ en Suisse, ni en Allemagne, ni en France, l'analyse n'a pas permis de conclure que les femmes en provenance de pays fortement marqués par le modèle patriarcal soient désavantagées. Au contraire même: en France, le taux de passage dans l'enseignement supérieur des femmes de cette origine est sensiblement plus élevé que celui des françaises. Par ailleurs, les résultats suggèrent que comparées à leurs pairs masculins, ces femmes ont même un avantage.

S'agissant des limites de la présente analyse, on ne peut ignorer d'éventuels effets d'association dans la mesure où - comme l'ont montré des modèles de validation appliqués à la France qui n'ont pas été présentés ici - certaines différences se décalent suivant les établissements de formation supérieure considérés. Compte tenu du degré élevé de stratification interne du système de formation français, il conviendrait également d'explorer si le taux de passage plus élevé des femmes dans les institutions du degré tertiaire pointe effectivement vers des carrières prestigieuses ou si elles constituent davantage une solution de repli. Notons encore que dans les pays étudiés, la part des divers pays d'origine varie d'une population migrante à l'autre. De plus, on ne saurait trop encourager les auteurs des prochaines recherches à étudier les mécanismes causals sous-tendant les effets secondaires de l'origine ethnique, probablement pas les mêmes pour les hommes et pour les femmes, mais également différents selon les conditions-cadre institutionnelles. Enfin, pour des analyses quantitatives envisageant d'examiner des groupes plus détaillés pour approfondir le concept d'intersectionnalité, il faudrait disposer de données portant sur un nombre de cas autrement plus élevé.

Pour résumer, on retiendra qu'il est profitable de prendre en considération les recoupements entre les axes d'inégalités (intersectionnalités). Du point 
de vue des sciences sociales, qui visent à formuler des généralisations, si l'on entend creuser ces intersectionnalités, il semble utile de trouver un équilibre entre l'attention portée au détail et les généralisations, ce qui n’ira pas sans poser quelques problèmes. S'agissant des études migratoires, il faut encore constater que la présente analyse des recoupements entre axes d'inégalités contredit l'image du migrant universellement désavantagé.

\section{Notes}

1 En Suisse: les titulaires d'une maturité, d'une maturité professionnelle, d'un diplôme (couronnant les études à l'école de culture générale), d'une maturité professionnelle; en Allemagne: Abitur, Abitur professionnel; en France: baccalauréat (excepté le baccalauréat professionnel).

2 Codé 1 en Suisse: universités, EPF, hautes écoles spécialisées et hautes écoles pédagogiques, en France: licence, master ou PhD, en Allemagne: universités, Fachhochschulen, Pädagogische Hochschulen et universités techniques.

3 Il serait certes souhaitable de décortiquer la situation par pays, mais les échantillons disponibles pour la Suisse ne permettaient pas d'affiner à ce point l'analyse. S'agissant de la première vague migratoire, nous avons donc formé des groupes dans lesquels la conception de la répartition des rôles entre hommes et femmes n'était pas la même (p. ex. ex-Yougoslavie $v$ s Turquie).

4 En Suisse, la catégorie «note manquante» est surreprésentée pour deux raisons: d'une part, parce que la part des données manquantes est particulièrement élevée $(33,4 \%)$, d'autre part, parce qu'à l'âge de 15 ans, les prestations PISA renvoient à des associations négatives.

5 Les résultats se rapportent à la deuxième génération de migrants, puisqu'ici encore, on a pris pour référence un effet d'interaction pour la conjugaison du sexe masculin et de l'appartenance à la première génération de migrants. L'effet correspondant est négatif, ce qui suggère, pour les hommes de cette deuxième génération, qu'ils afficheront, en termes relatifs, un taux de passage dans le supérieur plus bas.

\section{Bibliographie}

Algan, Y., Landais, C. \& Senik, C. (2010). Cultural Integration in France. Mimeo.

Arrow, K.J. (1973). The Theory of Discrimination. In O. Ashenfelter \& A. Rees (Ed.), Discrimination in Labour Markets (pp. 3-33). Princeton, NJ: Princeton University Press.

Arum, R., Gomoran, A. \& Shavit, Y. (2007). More Inclusion than Diversion. In Y. Shavit, R. Arum \& A. Gomoran (Ed.), Stratification in Higher Education (pp. 1-37). Stanford, CA: Stanford University Press.

Beck, M., Jäpel, J. \& Becker, R. (2010). Determinanten des Bildungserfolgs von Migranten im Schweizer Bildungssystem. In G. Quenzel \& K. Hurrelmann (Ed.), Bildungsverlierer Neue Ungleichheiten (S. 313-337). Wiesbaden: VS Verlag für Sozialwissenschaften.

Becker, G.S. (1957). The Economics of Discrimination. Chicago, IL: University of Chicago Press.

Becker, R. (2012). Der Übergang ins Hochschulstudium: Prozesse und Mechanismen am Beispiel der deutschen Schweiz. In M.M. Bergman, S. Hupka-Brunner, T. Meyer \& R. Samuel (Hrsg.), Bildung - Arbeit - Erwachsenwerden (pp. 305-331). Wiesbaden: Verlag für Sozialwissenschaften.

Becker, R. (2013). Bildung. Die wichtigste Investition in die Zukunft. In S. Hradil (Hrsg.), Deutsche Verhältnisse (S. 120-150). Frankfurt am Main: Campus.

Becker, R. \& Hecken, A.E. (2009a). Why are working-class children diverted from universities? European Sociological Revue, 25, 233-250. 
Becker, R. \& Hecken, A.E. (2009b). Higher Education or Vocational Training? An Empirical Test of the Rational Action Model of Educational Choices Suggested by Breen and Goldthorpe (1997) and Esser (1999). Acta Sociologica, 5,: 25-45.

Becker, R. \& Schubert, F. (2011). Die Rolle von primären und sekundären Herkunftseffekten für Bildungschancen von Migranten im deutschen Schulsystem. In R. Becker (Ed.), Integration durch Bildung. Bildungserwerb von jungen Migranten in Deutschland (S. 161-194). Wiesbaden: VS Verlag für Sozialwissenschaften.

Becker, R. \& Müller, W. (2011). Bildungsungleichheiten nach Geschlecht und Herkunft im Wandel. In R. Becker (Hrsg.), Geschlechtsspezifische Bildungsungleichheiten (S. 55-75). Wiesbaden: VS Verlag für Sozialwissenschaften.

Becker, R. \& Zangger, C. (2013). Die Bildungsexpansion in der Schweiz und ihre Folgen. Eine empirische Analyse des Wandels der Bildungsbeteiligung und -ungleichheiten mit den Daten der Schweizer Volkszählungen 1970, 1980, 1990 und 2000. Erscheint in Kölner Zeitschrift für Soziologie und Sozialpsychologie, 65.

BfS (Bundesamt für Statistik) (2013). Migration und Integration - Indikatoren. Bevölkerung mit Migrationshintergrund. http://www.bfs.admin.ch/bfs/portal/de/index/ themen/01/07/ blank/key/04. html (Accessed 28.05.2013).

bpb (Bundeszentrale für politische Bildung) (2012). Die soziale Situation in Deutschland. Bevölkerung mit Migrationshintergrund. http://www.bpb.de/nachschlagen/zahlen-undfakten/soziale-situation-in-deutschland/61646/migrationshintergrund-i. (Consulté le 28 mai 2013).

Boudon, R. (1974). Education, Opportunity and Social Inequality: Changing Prospects in Western Society. New York: Wiley.

Breen, R., Luijkx, R., Müller, W. \& Pollak, R. (2012). Bildungsdisparitäten nach sozialer Herkunft und Geschlecht im Wandel - Deutschland im internationalen Vergleich. In R. Becker \& H. Solga (Hrsg.), Soziologische Bildungsforschung (pp. 346-373). Wiesbaden: VS Verlag für Sozialwissenschaften.

Brinbaum, Y. \& Cebolla Boado, H. (2007). The School Careers of Ethnic Minority Youth in France: Success or Disillusion? Ethnicities, 7, 445-474.

British Council \& Migration Policy Group (2013). Migration Integration Policy Index (MIPEX). http://www.mipex.eu/countries. Accessed 18 January 2013.

Buchmann, C., DiPrete, T.A. \& McDaniel, A. (2008). Gender Inequalities in Education. Annual Review of Sociology, 34, 319-337.

Centre Maurice Halbwachs/Ministère de l'éducation nationale, DEPP (2011). Panel d'élèves du second degré, recrutement 1995 - France métropolitaine - 1995-2011. Description de l'enquête. Paris: CMH (http://www.cmh.greco.ens.fr/enquetes/XML/lil-0182.xml\#4).

CSRE (2010). Rapport sur l'éducation en Suisse 2010. Aarau: CSRE.

Diehl, C., Friedrich, M. \& Hall, A. (2009). Jugendliche ausländischer Herkunft beim Übergang in die Berufsausbildung: Vom Wollen, Können und Dürfen. Zeitschrift für Soziologie, 38, 48-67.

Duru-Bellat, M. \& Kieffer, A. (2008). From the Baccalaureat to Higher Education in France:Shifting Inequalities. Population (Edition anglaise), 63: 119-154.

Erikson, R. \& Jonsson, J.O. (1996). Explaining Class Inequality in Education: The Swedish Test Case. In R. Erikson \& J.O. Jonsson (Ed.), Can Education Be Equalized? The Swedish Case in Comparative Perspective (pp. 1-63). Stockholm: Westview Press.

Esser, H. (1999). Soziologie: Spezielle Grundlagen, Band 1: Situationslogik und Handeln. Frankfurt am Main: Campus.

Fibbi, R., Lerch, M. \& Wanner, P. (2006). Unemployment and Discrimination against Youth of Immigrant Origin in Switzerland: When the Name Makes the Difference. Journal of International Migration and Integration, 7, 351-366.

Fleischmann, F. \& Kristen, C. (2011). Gender inequalities in the education of the second generation. Mimeo. 
Ganzeboom, H.B.G., De Graaf, P.M. \& Treiman, D.J. (1992). A Standard International Socio-Economic Index of Occupational Status. Social Science Research, 21, 1-56.

Gresch, C. \& Kristen, C. (2011). Staatsbürgerschaft oder Migrationshintergrund? Ein Vergleich unterschiedlicher Operationalisierungsweisen am Beispiel der Bildungsbeteiligung. Zeitschrift für Soziologie, 40, 208-227.

Griga, D. (2014). Participation in Higher Education of Youths with a Migrant Background in Switzerland. Swiss Journal of Sociology, 40 (3), accepted.

Hadjar, A. \& Berger, J. (2011). Geschlechtsspezifische Bildungsungleichheiten in Europa: Die Bedeutung des Bildungs- und Wohlfahrtsstaatssystems. In A. Hadjar (Hrsg.), Geschlechtsspezifische Bildungsungleichheiten (S. 23-54). Wiesbaden: VS Verlag für Sozialwissenschaften.

Heath, A.F. \& Brinbaum, Y. (2007). Guest Editorial: Explaining ethnic inequalities in educational attainment. Ethnicities, 7, 291-305.

Imdorf, C. (2010). Wie Ausbildungsbetriebe soziale Ungleichheit reproduzieren: Der Ausschluss von Migrantenjugendlichen bei der Lehrlingsselektion. In H.-H. Krüger, U. Rabe-Kleberg, R.-T. Kramer \& J. Budde (Hrsg.), Bildungsungleichheit revisited. Bildung und soziale Ungleichheit vom Kindergarten bis zur Hochschule (S. 263-278). Wiesbaden: VS Verlag für Sozialwissenschaften.

Jackson, M., Jonsson, J.O. \& Rudolphi, F. (2012). Ethnic Inequality in Choice-driven Education Systems: A Longitudinal Study of Performance and Choice in England and Sweden. Sociology of Education, 85, 158-78.

Jonsson, J.O. \& Rudolphi, F. (2011). Weak Performance - Strong Determination: School Achievement and Educational Choice among Children of Immigrants in Sweden. European Sociological Review, 27, 487-508.

Kao, G. \& Tienda, M. (1995). Optimism and Achievement: The Educational Performance of Immigrant Youth. Social Science Quarterly, 76, 1-19.

Kao, G. \& Tienda, M. (1998). Educational Aspirations of Minority Youth. American Journal of Education, 106, 349-384.

Kristen, C. \& Granato, N. (2007). The Educational Attainment of the Second Generation in Germany. Social Origins and Ethnic Inequality. Ethnicities, 7, 343-366.

Kristen, C., Reimer, D. \& Kogan, I. (2008). Higher Education Entry of Turkish Immigrant Youth in Germany. International Journal of Comparative Sociology, 49, 127-151.

Lewis, J. \& Ostner, I. (1994). Gender and the Evolution of European Social Policies, Zentrum für Sozialpolitik der Universität Bremen, Arbeitspapier Nr. 4.

Lörz, M. \& Schindler, S. (2011). Geschlechtsspezifische Unterschiede beim Übergang ins Studium. In A. Hadjar (Hrsg.), Geschlechtsspezifische Bildungsungleichheiten (S. 99-122). Wiesbaden: VS Verlag für Sozialwissenschaften.

Luci, A. (2011). Frauen auf dem Arbeitsmarkt in Deutschland und Frankreich: Warum es Französinnen besser gelingt Familie und Beruf zu vereinbaren. Berlin: Friedrich-EbertStiftung.

Maurice, M., Sellier, F. \&, Silvestre, J.-J. (1982). Politique d'éducation et d'organisation industrielle en France et en Allemange: Essai d'analyse sociétal. Paris: PUF.

Meyer, T. (2003). Jugendliche mit Migrationshintergrund. In BfS \& TREE (Hrsg.), Wege in die nachobligatorische Ausbildung (S. 111-118). Neuchâtel: Bundesamt für Statistik.

Müller, W. \& Pollak, R. (2007). Weshalb gibt es so wenige Arbeiterkinder in Deutschlands Universitäten? In R. Becker \& W. Lauterbach (Hrsg.), Bildung als Privileg (S. 303-342). Wiesbaden: VS Verlag für Sozialwissenschaften.

Müller, W. \& Shavit, Y. (1998). The Institutional Embeddedness of the Stratification Process: A Comparative Study of Qualifications and Occupations in Thirteen Countries. In Y. Shavit \& W. Müller (Ed.), From School to Work (pp. 1-48). Oxford: Clarendon Press.

Phelps, E.S. (1972). The Statistical Theory of Racism and Sexism. American Economic Review, 62: 659-661. 
Relikowski, I., Yilmaz, E. \& Blossfeld, H.-P. (2012). Wie lassen sich die hohen Bildungsaspirationen von Migranten erklären? In R. Becker \& H. Solga (Hrsg.) Soziologische Bildungsforschung (S. 111-136). Wiesbaden: VS Verlag für Sozialwissenschaften.

Sacchi, S. (2011). TREE-Längsschnittgewichtung: Konstruktion und Anwendung, Dokumentation zu den acht Erhebungswellen 2000 bis 2007. mimeo.

Simon, P. (2003). France and the Unknown Second Generation: Preliminary Results on Social Mobility. International Migration Review, 37, 1091-1119.

Solga, H. \& Becker, R. (2012). Soziologische Bildungsforschung - eine kritische Bestandsaufnahme. In Y. Shavit \& W. Müller (Hrsg.) Soziologische Bildungsforschung. Sonderheft 52 der Kölner Zeitschrift für Soziologie und Sozialpsychologie (S. 7-43). Wiesbaden: VS Verlag für Sozialwissenschaften.

TREE (Ed.) (2010). Projekt-Dokumentation 2000-2007. Basel: TREE.

Vallet, L.-A. (2005). What Can We Do to Improve the Education of Children from Disadvantaged Backgrounds? Joint Working Group on Globalization and Education of the Pontifical Academy of Sciences and the Pontifical Acadamy of Social Sciences. Casino Pio IV, Vatican City.

Vallet, L.-A. \& Caille, J.-P. (1996). Les élèves étrangers ou issus de l'immigration dans l'école et le collège français. Les dossiers d'Education et Formations 67. Paris: Direction de l'Evaluation et de la Prospective, Ministère de l'Education nationale.

Vallet, L.-A. \& Caille, J.-P. (1999). Migration and Integration in France. Academic Careers of Immigrants' Children in Lower and Upper Secondary School. Paper prepared for the ESF Conference "European Societies or European Society? Migrations and Inter-Ethnic Relations in Europe" Obernai, September 23-28 1999. Session "Migrants and Educational Success". Mimeo.

Van de Werfhorst, H.G. \& Van Tubergen, F. (2007). Ethnicity, Schooling, and Merit in the Netherlands. Ethnicities, 3, 416-444.

Walby, S., Armstrong, J. \& Strid, S. (2012). Intersectionality: Multiple Inequalities in Social Theory. Sociology, 46, 224-240.

Wooldridge, J.M. (2002). Econometric Analysis of Cross Section and Panel Data. Cambridge, MA: MIT Press.

Mots-clés: Inégalités en matière d'éducation, genre, contexte migratoire, formation supérieure, comparaison internationale 


\section{Bildungsungleichheiten beim Hochschulzugang nach Geschlecht und Migrationshintergrund: Befunde aus der Schweiz, Deutschland und Frankreich}

\section{Zusammenfassung}

Im Rahmen des Beitrags wird der Übergang ins Hochschulsystem unter besonderer Berücksichtigung der Verschränkung von geschlechts- und migrationsbezogenen Bildungsungleichheiten betrachtet. Im Zentrum der auf entscheidungstheoretischen Überlegungen beruhenden Analysen steht ein Vergleich zwischen der Schweiz (TREE), Deutschland (HIS-Studienberechtigte 2002) und Frankreich (Panel d'élèves du second degré 1995). In den Resultaten zeigen sich unter Kontrolle der sozialen Herkunft für die Schweiz und für Deutschland erhöhte Übergangsraten ins Hochschulsystem für Männer aus einigen der sozial benachteiligten Migrantengruppen (2. Generation). Belege für eine Benachteiligung von Migrantinnen der zweiten Generation aus eher patriarchalisch geprägten Herkunftsländern finden sich in keinem der analysierten Länder.

Schlagworte: Bildungsungleichheit, Geschlecht, Migrationshintergrund, Hochschulbildung, internationaler Vergleich

\section{Diseguaglianze educative rispetto al genere e al background migratorio nell'accesso alla formazione superiore. Risultati da Svizzera, Germania e Francia.}

\section{Riassunto}

Questo articolo tratta della transizione alla formazione superiore mettendo a tema le diseguaglianze educative all'intersezione tra genere e passato migratorio. $\mathrm{Al}$ cuore di queste analisi, condotte entro il quadro di riferimento della teoria delle decisioni, si trova un confronto tra Svizzera (TREE), Germania (HIS-Studienberechtigte 2002) e Francia (Panel d'élèves du second degré 1995). I risultati mostrano un aumento della quota di studenti che accedono alla formazione superiore per I maschi di seconda generazione di alcuni gruppi svantaggiati di migranti in Svizzera e Germania, operando un controllo sull'origine sociale. I dati, per i paesi considerati, non confermano uno svantaggio per le ragazze di seconda generazione che hanno origini in paesi caratterizzati da una cultura patriarcale.

Parole chiave: Diseguaglianze educative, genere, background migratorio, formazione superiore, confronto internazionale. 


\section{Educational inequalities related to gender and migrant background at the transition to Higher Education. Findings from Switzerland, Germany and France}

Summary

The paper deals with the transition to Higher Education focusing on educational inequalities at the intersection of gender and migrant background. At the core of the analyses, theorized referring to decision theory, is a comparison of Switzerland (TREE), Germany (HIS-Studienberechtigte 2002) and France (Panel d'élèves du second degré 1995). Results indicate increased transition rates to Higher Education for second-generation males from some socially disadvantaged migrant groups for Switzerland and Germany, if controlled for social origin. Evidence confirming a female disadvantage for second generation females originating from countries characterised by a rather patriarchal culture were not found in any of the countries under consideration.

Key words: Educational inequality, gender, migrant background, Higher Education, international comparison 
